\title{
A New Pricing Scheme for Controlling Energy Storage Devices in Future Smart Grid
}

\author{
Jingwei Zhu, ${ }^{1}$ Michael Z. Q. Chen, ${ }^{2}$ and Baozhu $\mathrm{Du}^{3}$ \\ ${ }^{1}$ Department of Mechanical Science and Engineering, University of Illinois at Urbana-Champaign, IL 61801, USA \\ ${ }^{2}$ Department of Mechanical Engineering, The University of Hong Kong, Hong Kong \\ ${ }^{3}$ School of Automation, Nanjing University of Science and Technology, Nanjing 210094, China
}

Correspondence should be addressed to Baozhu Du; dubaozhu@gmail.com

Received 25 June 2014; Accepted 1 August 2014; Published 3 September 2014

Academic Editor: Zhiwei Gao

Copyright (C) 2014 Jingwei Zhu et al. This is an open access article distributed under the Creative Commons Attribution License, which permits unrestricted use, distribution, and reproduction in any medium, provided the original work is properly cited.

\begin{abstract}
Improvement of the overall efficiency of energy infrastructure is one of the main anticipated benefits of the deployment of smart grid technology. Advancement in energy storage technology and two-way communication in the electric network are indispensable components to achieve such a vision, while efficient pricing schemes and appropriate storage management are also essential. In this paper, we propose a universal pricing scheme which permits one to indirectly control the energy storage devices in the grid to achieve a more desirable aggregate demand profile that meets a particular target of the grid operator such as energy generation cost minimization and carbon emission reduction. Such a pricing scheme can potentially be applied to control the behavior of energy storage devices installed for integration of intermittent renewable energy sources that have permission to grid connection and will have broader applications as an increasing number of novel and low-cost energy storage technologies emerge.
\end{abstract}

\section{Introduction}

In recent years, there has been growing interest in the development of intelligent electricity network technologies, collectively called the smart grid, which meet the needs for future energy provision [1-9]. It is now a very active research topic in the area of modeling and control of complex dynamic systems $[10,11]$. A smarter grid is expected to make the grid work far more efficiently by applying tools and technologies available now [2]. It will fully accommodate renewable and traditional energy sources, potentially reducing carbon footprint and improving efficiencies.

Renewable energy sources such as photovoltaic solar systems and wind turbines will have growing importance in future power generation systems. However, exploitation of renewable energy resources can be problematic as renewable power generation is usually intermittent and variable. Therefore, energy storage systems are increasingly being used to help integrate renewable power generation into the grid [12-16]. For instance, some battery energy storage systems are capable of absorbing and delivering both real and reactive power with subsecond response times, which mitigates the adverse effect on system stability due to the introduction of renewable power. And different energy storage technologies-for example, pumped-hydroenergy storage, electrochemical energy storage, and supercapacitorcan be combined in order to give full play to their own characteristics and advantages. Also, energy storage control systems can be integrated with energy markets to make more economical use of energy. The purpose is to lower the peak load, which requires the support of expensive and also carbon intensive peaking power plant generators, so that both carbon emissions and energy generation costs are lowered. The end users will definitely benefit as electricity price decreases.

It is still at too early a stage for widespread adoption of small-scale consumer storage devices, even though the potential has been foreseen $[4,17,18]$. And cost-effectiveness of energy storage as an arbitrage instrument depends on capital costs, operations, and maintenance costs as well as price incentives from the grid. In most cases up to now, energy arbitrage as a sole revenue source does not appear to be economically viable. Additional high-value ancillary 
services such as smoothing the volatile power output and voltage regulation need to be bundled $[14,19]$, while at the same time, more attractive and efficient pricing schemes have to be provided by the grid [16].

In this paper, we focus on the pricing scheme set by grid owners and operators, which indirectly controls energy storage devices in the grid. There are many pricing schemes available in the smart grid literature $[4,18,20-23]$, most of which assume that users or other agents such as energy storage devices in the grid are all self-interested and try to minimize their payment to grid or maximize their income. Mohsenian-Rad et al.s billing model in [20, 21] assumes that users are charged proportionally to their daily energy consumption and total daily charges to the users are proportional to total daily energy generation costs. This model does not welcome the introduction of energy storage devices since they always increase energy consumption. And shift of load from peak to off-peak periods brings little immediate gains to load shifters themselves although it benefits the grid and other users, which implies share of interest. In $[18,22]$, price of electricity at certain time interval depends on aggregate demands in the grid at that time interval. Since aggregate demand profile in the coming day cannot be known in advance, prediction of market prices is needed for demand side management. In [4], Voice et al. propose that at the end of each day price profile for the coming day based on current loads is announced so that energy storage devices do not need to speculate on future prices in order to optimize their storage profile in terms of income maximization in the coming day. As explicit incentives are provided by the pricing function, a damping term is added to the bill to ensure stability. It is proved that, under this pricing scheme with some strictly increasing differentiable pricing function, aggregate demand profile converges to a unique equilibrium. A specific example of the pricing scheme is also provided with pricing functions designed to recover supplier costs. The behavior of energy storage devices in the grid under this model is more predictable and controllable for the grid operator. Our pricing scheme adopts the same mechanism.

We propose a universal pricing scheme for controlling energy storage devices in the grid, which also takes integration of renewable energy into consideration. It guarantees convergence to the optimal aggregate demand profile which minimizes the convex objective function defined by grid operators when user load and renewable energy generation profile keep constant and each energy storage device is operated optimally in terms of income maximization. In the situation where user load and renewable energy generation change from day to day, it can still efficiently reduce the value of the objective function, which can satisfactorily meet a particular target of grid operators. This pricing scheme can be applied to energy storage devices installed for integration of intermittent renewable energy with permission to grid connection. They are more economically feasible at current stage as they are used for multiple functions. And as an increasing number of novel and low-cost energy storage technologies emerge, which will possibly justify the use of either large-scale or small-scale consumer energy storage as an arbitrage instrument, our pricing scheme will have much broader applications in the future.

\section{Model Description}

This section describes the model used. Consider a smart power system which contains several users and energy storage devices. We are interested in the storage management during the time period $\mathscr{H}=[1, H]$. Without loss of generality, we can assume that time granularity is one hour and $H=24$.

2.1. User. Let $\mathcal{N}=\{1, \ldots, N\}$ denote the set of users and let $x_{n}^{h}$ denote user $n$ 's load during time slot $h$. Our pricing scheme is only applied to energy storage devices that have permission to grid connection. Users can be charged according to other simpler pricing schemes such as flat pricing or peak load pricing and control of their load profile is not discussed in this paper.

2.2. Energy Storage Device. Let $\mathscr{M}=\{1, \ldots, M\}$ denote the set of energy storage devices. Assume that they are all self-interested and try to minimize their own payment or maximize the income. Each energy storage device $m$ has a capacity of $e_{m}$, charge efficiency of $a_{m}<1$, and discharge efficiency of $b_{m}<1$. If $q$ amount of energy is consumed to charge the device, only $a_{m} q$ can be stored. Similarly, if $q$ amount of energy is stored, only $b_{m} q$ can be discharged. Let $s_{m}^{h}$ denote the storage profile of $m$. We have $s_{m}^{h}=s_{m}^{h+}-s_{m}^{h-}$, $s_{m}^{h+} \cdot s_{m}^{h-}=0$, for all $h \in \mathscr{H}$, where $s_{m}^{h+}$ is the charging profile and $s_{m}^{h-}$ is the discharging profile. $0 \leq s_{m}^{h+} \leq s_{+}, 0 \leq$ $s_{m}^{h-} \leq s_{-}$, for all $h \in \mathscr{H}$, where $s_{-}$is the discharging volume and $s_{+}$is the charging volume of the device for one time interval. Let $v_{m}^{h}$ denote possible energy generation from the renewable energy sources connected with device $m$ at time slot $h$. Renewable energy can be stored into energy storage devices for a later sale or sold to the grid directly. Assume that energy storage in each device at the end of each day comes back to the same level as the beginning of the day, $a b \sum_{h=1}^{H} s_{m}^{h+}=\sum_{h=1}^{H} s_{m}^{h-}$. Apparently $\sum_{h=1}^{H} s_{m}^{h} \geq 0$. Moreover, energy that can be stored or discharged at time slot $h$ satisfies $s_{m}^{h-} / b_{m} \leq e_{m}^{0}+\sum_{j=1}^{h-1}\left(a_{m} s_{m}^{j+}-s_{m}^{j-} / b_{m}\right), a_{m} s_{m}^{h+} \leq e_{m}-e_{m}^{0}-$ $\sum_{j=1}^{h-1}\left(a_{m} s_{m}^{j+}-s_{m}^{j-} / b_{m}\right)$, for all $h \in \mathscr{H}$, where $e_{m}^{0}$ is the initial energy storage at the beginning of $\mathscr{H}$. Let $\mathcal{S}_{m}$ represent the set of valid storage profiles for $m$, and set $\mathcal{S}=\times_{m \in \mathscr{M}} \mathcal{S}_{m}$ where $x$ denotes the Cartesian product of vector spaces. The true energy exchange profile between energy storage device and grid is $S_{m}^{h}=s_{m}^{h}-v_{m}^{h}$.

\section{Pricing Scheme}

Let $l^{h}$ denote the aggregate demand in the grid at time slot $h$ and by definition $l^{h}=\sum_{m \in \mathscr{M}} S_{m}^{h}+\sum_{n \in \mathscr{N}} x_{n}^{h}$, for all $h \in \mathscr{H}$. Grid operators usually have particular targets for aggregate demand profile. One common design objective in a power distribution system is energy generation cost minimization: 
minimize $_{s \in \mathcal{S}} \sum_{h=1}^{H} C^{h}\left(l^{h}\right)$. Cost function $C^{h}$ is assumed to be strictly increasing and convex. Usually, we have $C^{h}(L)=$ $a_{k} L^{2}+b_{k} L+c_{k}$, where $a_{k}>0$ and $b_{k}, c_{k} \geq 0$ are predetermined parameters. According to the target and objective function, grid operators can adjust pricing scheme to steer energy storage devices in the grid. Our work in this paper mainly focuses on finding the most efficient pricing scheme, under which the convex objective function is minimized when each energy storage device strives to maximize their income.

Assume that the grid operator announces the pricing scheme for the next day at the end of each day. Under this assumption, energy storage devices do not need to make predictions on future market prices in order to optimize their storage profile. And they are allowed to sell electricity to the grid at the same price as the grid sells electricity according to the pricing scheme announced.

\subsection{Constant User Load Profile and Renewable Energy Genera-} tion. We first consider a situation where the user load profile is constant (user load profile may vary a little from day to day if there is no sudden weather change taking place or other events which may change user behavior significantly) and so is the renewable power generation. Define a pricing function $p^{h}$ indicating the price for electricity at time slot $h \in \mathscr{H}$ set by the grid operator. Consider the situation where the grid operator announces the price $p^{h}$ for each $h$ of the coming day. As energy storage devices in the grid all react to the same price signals in the way that their income is maximized, the aggregate behavior can be unstable.

In [4], Voice et al. propose a pricing mechanism which introduces a damping term to guarantee stability. That is, each energy storage device $m \in \mathscr{M}$ is charged an additional fee of $\sum_{h=1}^{H} \mathscr{K}\left(s_{m}^{h}-\widehat{s}_{m}^{h}\right)^{2}$, where $\widehat{s}_{m}^{h}$ is the storage profile of the day before and $\mathscr{K}>0$. We employ the same mechanism in our pricing scheme when the objective function takes the form of $\sum_{h=1}^{H} C^{h}\left(l^{h}\right)=\sum_{h=1}^{H}\left(a_{k} l^{h^{2}}+b_{k} l^{h}+c_{k}\right)$, where $a_{k}>0$ and $b_{k}, c_{k} \geq$ 0 . For each energy storage device $m \in \mathscr{M}$, let $B_{m}$ denote the amount to be charged for $\mathscr{H}$. If $B_{m}<0$, device $m$ earns revenue through the daily operation. At the beginning of each day, every device $m$ makes optimal decision on its storage profile which yields to all the constraints mentioned before using convex optimization methods so that the aggregate income in the coming day is maximized.

We propose that, at the end of each day, pricing scheme for the next day is announced and

$$
B_{m}=\sum_{h=1}^{H} S_{m}^{h} p^{h}+\mathscr{K}\left(s_{m}^{h}-\widehat{s}_{m}^{h}\right)^{2}
$$

where $B_{m}$ is the amount to be charged in the coming day, $p^{h} /\left(2 a_{k} \bar{l}^{h}+b_{k}\right)=\mathscr{K} / a_{k} M=c>0, c$ is a constant set by grid operators to adjust the ratio of arbitrage benefit to grid benefit and has no influence on storage profile, $a_{k}, b_{k}$ come from the objective function $\sum_{h=1}^{H} C^{h}\left(l^{h}\right)=\sum_{h=1}^{H}\left(a_{k} l^{h^{2}}+b_{k} l^{h}+c_{k}\right), M$ is the total number of energy storage devices, and $\tilde{l}^{h}$ is the aggregate demand profile in the day before.
It can be proved that, with such a pricing scheme, the objective function is nonincreasing from day to day if all the energy storage devices are operated optimally in terms of income maximization.

Theorem 1. Given objective function $\sum_{h=1}^{H} C^{h}\left(l^{h}\right)=$ $\sum_{h=1}^{H}\left(a_{k} l^{h^{2}}+b_{k} l^{h}+c_{k}\right)$, where $a_{k}>0$ and $b_{k}, c_{k} \geq 0$, the objective function is nonincreasing if pricing scheme (1) is applied and each energy storage device $m \in \mathscr{M}$ adopts the following optimal storage profile $s_{m}=\operatorname{argmin}_{s_{m} \in \delta_{m}} B_{m}$.

It is reasonable to expect that $l^{h}>0$, for all $h \in \mathscr{H}$ and $C^{h}\left(l^{h}\right)>0$. Therefore, $\sum_{h=1}^{H} C^{h}\left(l^{h}\right)$ is lower bounded. Since it is nonincreasing from day to day, we may conclude that the objective function and storage profile of each device $m \in \mathscr{M}$ will all converge to an equilibrium.

The optimal storage profile solution to the objective function minimization problem and the minimum objective function value can be achieved in a centralized manner with convex optimization algorithm such as Interior Point Method with all the parameters known. We then prove that, under our pricing scheme, the objective function will converge to the minimum value calculated centrally.

Theorem 2. Given objective function $\sum_{h=1}^{H} C^{h}\left(l^{h}\right)=$ $\sum_{h=1}^{H}\left(a_{k} l^{h^{2}}+b_{k} l^{h}+c_{k}\right)$ where $a_{k}>0$ and $b_{k}, c_{k} \geq 0$, the objective function converges to $\min _{s \in \mathcal{S}} \sum_{h=1}^{H} C^{h}\left(l^{h}\right)$ if pricing scheme (1) is applied and each energy storage device $m \in \mathscr{M}$ adopts the following optimal storage profile $s_{m}=\operatorname{argmin}_{s_{m} \in \mathcal{S}_{m}} B_{m}$.

The pricing scheme can be further generalized for other convex objective functions. For grid operators, they may first approximate their own objective function by using a finite number of terms. For example, the function

$$
C^{h}\left(l^{h}\right)= \begin{cases}1 \times 10^{5}+60 l^{h}, & 0<l^{h}<5000 \\ -2 \times 10^{5}+120 l^{h}, & 5000 \leq l^{h}<8000\end{cases}
$$

shown in Figure 1 can be approximated as $C^{h}\left(l^{h}\right) \approx 8.7264 \times$ $10^{-7} l^{h^{3}}-0.0043 l^{h^{2}}+63.4167 l^{h}+1.0101 \times 10^{5}, 0<l^{h}<8000$, by polynomial curve fitting.

Then, more generally, $\sum_{h=1}^{H} C^{h}\left(\widetilde{l}^{h}+\Delta l^{h}\right)-C^{h}\left(\widetilde{l}^{h}\right)$ takes the form of $\sum_{h=1}^{H} A_{1}^{h} \Delta l^{h}+A_{2}^{h} \Delta l^{h^{2}}+A_{3}^{h} \Delta l^{h^{3}}+A_{4}^{h} \Delta l^{h^{4}}+\cdots$. Consider

$$
\begin{aligned}
& \sum_{h=1}^{H} C^{h}\left(\widetilde{l}^{h}+\Delta l^{h}\right)-C^{h}\left(\widetilde{l}^{h}\right) \\
& \quad \leq \sum_{h=1}^{H} A_{1}^{h} \Delta l^{h}+A_{2}^{h} P_{2}\left(A_{2}^{h}, \Delta l^{h}\right) \\
& \quad+A_{3}^{h} Q_{3}\left(A_{3}^{h}, \Delta l^{h}\right)+A_{4}^{h} P_{4}\left(A_{4}^{h}, \Delta l^{h}\right)+\cdots,
\end{aligned}
$$




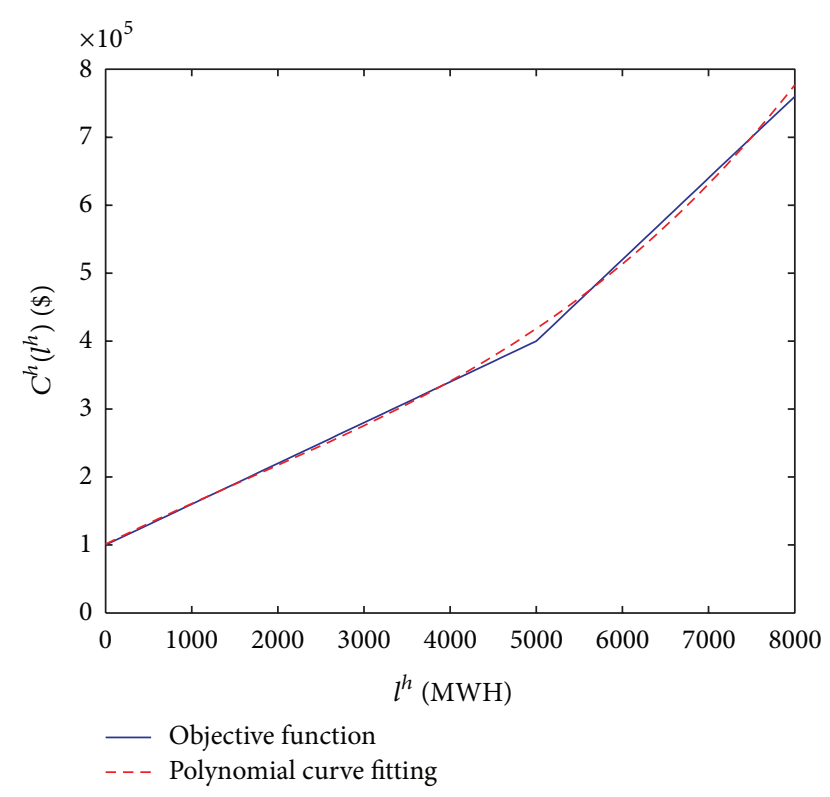

FIGURE 1: Objective function and corresponding polynomial curve fitting.

where

$$
\begin{aligned}
P_{n}\left(A_{n}^{h}, L\right) & =\left\{\begin{array}{ll}
L^{n}, & A_{n}^{h} \geq 0 ; \\
0, & A_{n}^{h}<0 .
\end{array} \quad n>0, n\right. \text { is even, } \\
Q_{n}\left(A_{n}^{h}, L\right) & =\left\{\begin{array}{ll}
0, & L<0, A_{n}^{h} \geq 0 ; \\
L^{n}, & L \geq 0, A_{n}^{h} \geq 0 ; \\
0, & L \geq 0, A_{n}^{h}<0 ; \\
L^{n}, & L<0, A_{n}^{h}<0 .
\end{array} \quad n>1, n\right. \text { is odd. }
\end{aligned}
$$

Figure 2 shows some examples of $P_{n}\left(A_{n}^{h}, L\right)$ and $Q_{n}\left(A_{n}^{h}, L\right)$ with comparison to $L^{n}$. Note that $A_{n}^{h} P_{n}\left(A_{n}^{h}, L\right)$ and $A_{n}^{h} Q_{n}\left(A_{n}^{h}, L\right)$ are all convex.

The universal pricing scheme should be

$$
\begin{aligned}
B_{m}=\sum_{h=1}^{H} S_{m}^{h} p^{h}+\mathscr{K}_{1}^{h} P_{2}\left(A_{2}^{h}, s_{m}^{h}-\widehat{s}_{m}^{h}\right) \\
+\mathscr{K}_{2}^{h} Q_{3}\left(A_{3}^{h}, s_{m}^{h}-\widehat{s}_{m}^{h}\right) \\
+\mathscr{K}_{3}^{h} P_{4}\left(A_{4}^{h}, s_{m}^{h}-\widehat{s}_{m}^{h}\right)+\cdots,
\end{aligned}
$$

where $p^{h} / A_{1}^{h}=\mathscr{K}_{1}^{h} / A_{2}^{h} M=\mathscr{K}_{2}^{h} / A_{3}^{h} M^{2}=\mathscr{K}_{3}^{h} / A_{4}^{h} M^{3}=$ $\cdots=\mathscr{K}_{n}^{h} / A_{n+1}^{h} M^{n}=c>0$ and $M$ is the total number of energy storage devices in the grid. Constant $c$ is set by grid operators to adjust the ratio of arbitrage benefit to grid benefit and has no influence on storage profile.

We then prove that, with such a pricing scheme, the convex objective function is nonincreasing from day to day if all the energy storage devices are operated optimally in terms of income maximization.
Theorem 3. Given convex objective function $\sum_{h=1}^{H} C^{h}\left(l^{h}\right)$, the objective function is nonincreasing if pricing scheme (5) is applied and each energy storage device $m \in \mathscr{M}$ adopts the following optimal storage profile $s_{m}=\operatorname{argmin}_{s_{m} \in \mathcal{S}_{m}} B_{m}$.

Similarly, the convex objective function $\sum_{h=1}^{H} C^{h}\left(l^{h}\right)$ converges to $\min _{s \in \mathcal{S}} \sum_{h=1}^{H} C^{h}\left(l^{h}\right)$. The proof is omitted here.

3.2. Changing User Load Profile and Renewable Energy Generation Connection. For the situation where user load profile and renewable energy generation change from day to day, we can slightly revise the pricing scheme introduced previously to accommodate the changes. Assume that grid operators and energy storage devices have perfect prediction, respectively, on the total user load profile $X^{h}=\sum_{n \in \mathcal{N}} x_{n}^{h}$ and renewable energy generation $v_{m}^{h}$ in the coming day. That is, perfect prediction for the next day on total user load profile $\dot{X}^{h}$ or renewable energy generation $\hat{v}_{m}^{h}$ is achieved at the end of each day. Each device will send its prediction $\hat{v}_{m}^{h}$ to grid operator, which will be used as part of the pricing scheme later.

For objective function $\sum_{h=1}^{H} C^{h}\left(l^{h}\right)=\sum_{h=1}^{H}\left(a_{k} l^{2}+b_{k} l^{h}+\right.$ $c_{k}$ ), where $a_{k}>0$ and $b_{k}, c_{k} \geq 0$, we change the pricing scheme to

$$
B_{m}=\sum_{h=1}^{H} S_{m}^{h} p^{h}+\mathscr{K}\left(s_{m}^{h}-\widehat{s}_{m}^{h}\right)^{2},
$$

where $p^{h} /\left\{2 a_{k}\left[\dot{X}^{h}+\sum_{m \in \mathscr{M}}\left(\widehat{s}_{m}^{h}-\hat{v}_{m}^{h}\right)\right]+b_{k}\right\}=\mathscr{K} / a_{k} M=c>0$ and $\bar{s}_{m}^{h}$ is storage profile of $m$ in the day before.

In the situation where user load and renewable power profile are constant, we actually make prediction that user load and renewable generation in the coming day will keep the same as in the previous days. Thus to accommodate the changes, we need to replace $\tilde{l}^{h}$ with $\dot{X}^{h}+\sum_{m \in M}\left(\widehat{s}_{m}^{h}-\hat{v}_{m}^{h}\right)$.

Since each energy storage device $m$ is assumed to be operated optimally in terms of income maximization, if $\widetilde{s}_{m}+\Delta s_{m}$ is adopted as storage profile of next day, $B_{m}\left(\widetilde{s}_{m}+\right.$ $\left.\Delta s_{m}, \hat{v}_{m}^{h}\right)-B_{m}\left(\widetilde{s}_{m}, \hat{v}_{m}^{h}\right) \leq 0$. It is easy to show that

$$
\begin{array}{r}
\sum_{h=1}^{H} C^{h}\left[\dot{X}^{h}+\sum_{m \in \mathscr{M}}\left(\widehat{s}_{m}^{h}-\hat{v}_{m}^{h}+\Delta s_{m}^{h}\right)\right] \\
-C^{h}\left[\dot{X}^{h}+\sum_{m \in \mathscr{M}}\left(\widehat{s}_{m}^{h}-\hat{v}_{m}^{h}\right)\right] \leq 0,
\end{array}
$$

where $\dot{X}^{h}+\sum_{m \in \mathscr{M}}\left(\hat{s}_{m}^{h}-\hat{v}_{m}^{h}+\Delta s_{m}^{h}\right)$ is exactly the aggregate demand profile of the coming day if all the predictions are accurate.

Therefore, under the control of our pricing scheme, the value of the objective function can always be reduced or kept the same when optimal aggregate demand profile is reached by the changes of storage profile made according to price signals compared with the situation where no changes of storage profile are made, if perfect predictions of total user load and renewable energy generation together with optimal operation of storage devices are assumed. In most cases the 


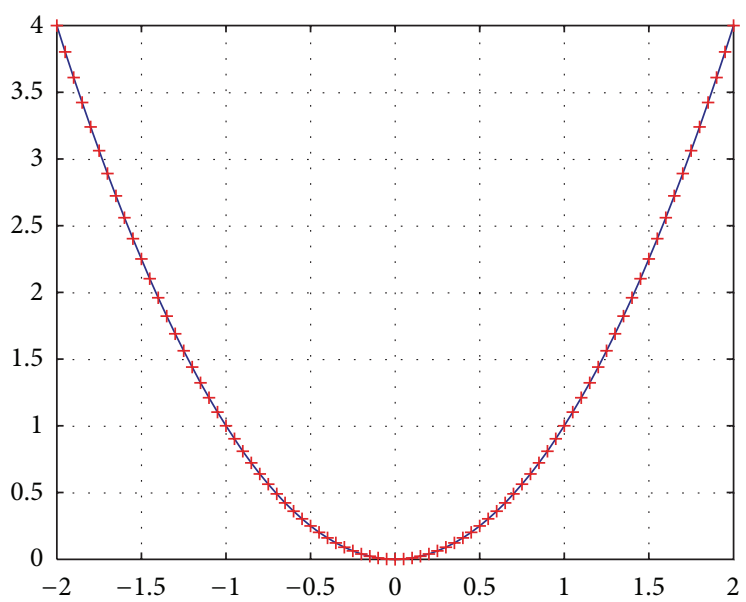

(a)

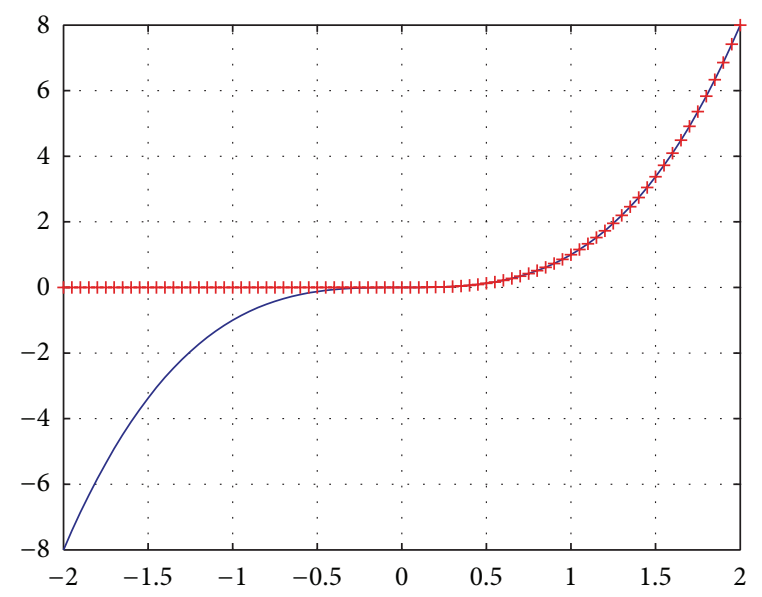

(c)

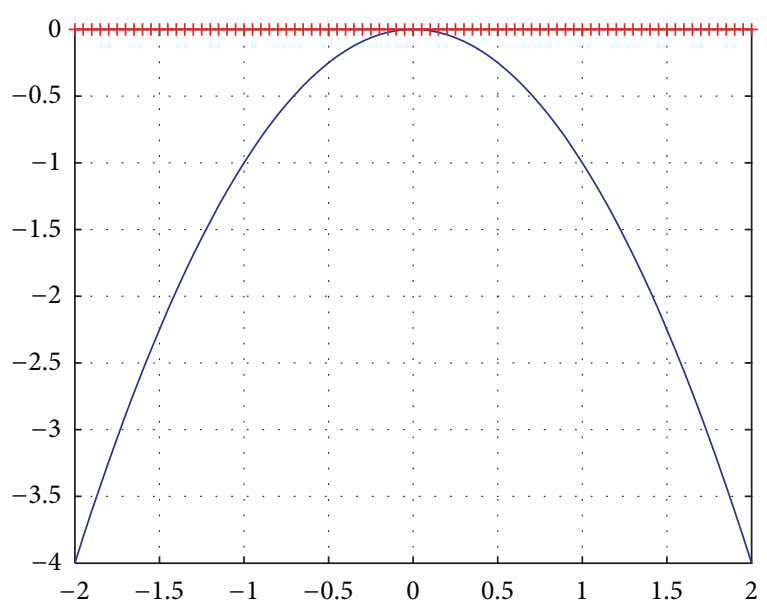

(b)

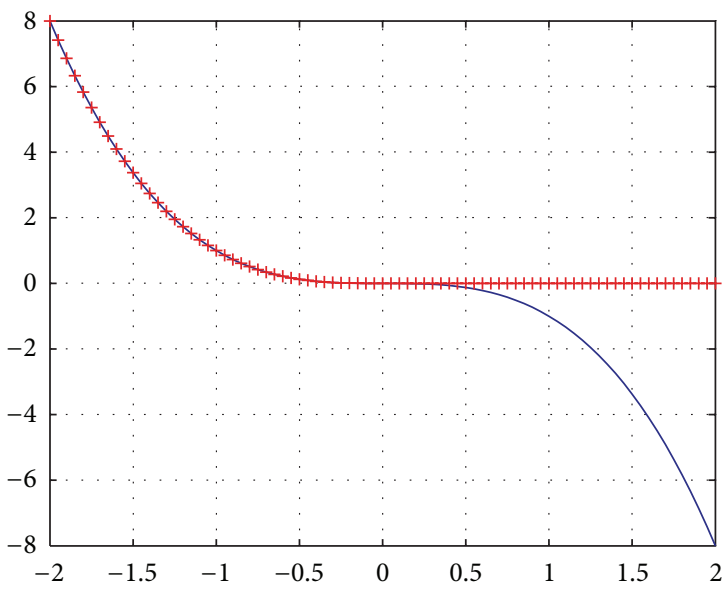

(d)

Figure 2: $P_{n}\left(A_{n}^{h}, L\right)$ and $Q_{n}\left(A_{n}^{h}, L\right)$ compared with $L^{n}$ : (a) $P_{2}\left(A_{2}^{h}, L\right)$ and $L^{2}$, where $A_{2}^{h} \geq 0$. (b) $-P_{2}\left(A_{2}^{h}, L\right)$ and $-L^{2}$, where $A_{2}^{h}<0$. (c) $Q_{3}\left(A_{3}^{h}, L\right)$ and $L^{3}$, where $A_{3}^{h} \geq 0$. (d) $-Q_{3}\left(A_{3}^{h}, L\right)$ and $-L^{3}$, where $A_{3}^{h}<0$.

better the prediction made by grid operators on next day total user load profile, the lower the value of objective function which can be achieved. However under this pricing scheme, energy storage device operators have no incentive to make efforts for accurate prediction of renewable power generation profile in the coming day. Thus, our pricing scheme can be further revised to

$$
\begin{gathered}
B_{m}=\sum_{h=1}^{H} S_{m}^{h} \frac{\mathscr{K}\left\{2 a_{k}\left[\dot{X}^{h}+\sum_{m \in \mathscr{M}}\left(\hat{s}_{m}^{h}-\hat{v}_{m}^{h}\right)\right]+b_{k}\right\}}{a_{k} M} \\
+\mathscr{K}\left(s_{m}^{h}-\widehat{s}_{m}^{h}\right)^{2}+\mathscr{J}\left(v_{m}^{h}-\hat{v}_{m}^{h}\right)^{2},
\end{gathered}
$$

where $\left(v_{m}^{h}-\hat{v}_{m}^{h}\right)$ is the difference between the true renewable power generation and the predicted renewable power generation, $\mathscr{J}>0$, and $\mathscr{J}\left(v_{m}^{h}-\hat{v}_{m}^{h}\right)^{2}$ provides the incentive for more accurate prediction.
For more general convex objective functions,

$$
\begin{gathered}
\sum_{h=1}^{H} C^{h}\left[\dot{X}^{h}+\sum_{m \in \mathscr{M}}\left(\vec{s}_{m}^{h}-\hat{v}_{m}^{h}+\Delta s_{m}^{h}\right)\right] \\
-C^{h}\left[\dot{X}^{h}+\sum_{m \in \mathscr{M}}\left(\hat{s}_{m}^{h}-\hat{v}_{m}^{h}\right)\right]
\end{gathered}
$$

takes the form of

$$
\begin{aligned}
& \sum_{h=1}^{H} A_{1}^{h^{\prime}} \sum_{m \in \mathscr{M}} \Delta s_{m}^{h}+A_{2}^{h^{\prime}}\left(\sum_{m \in \mathscr{M}} \Delta s_{m}^{h}\right)^{2} \\
& \quad+A_{3}^{h^{\prime}}\left(\sum_{m \in \mathscr{M}} \Delta s_{m}^{h}\right)^{3}+A_{4}^{h^{\prime}}\left(\sum_{m \in \mathscr{M}} \Delta s_{m}^{h}\right)^{4}+\cdots .
\end{aligned}
$$


Similarly, to ensure that $\sum_{h=1}^{H} C^{h}\left[\hat{X}^{h}+\sum_{m \in M}\left(\widehat{s}_{m}^{h}-\hat{v}_{m}^{h}+\right.\right.$ $\left.\left.\Delta s_{m}^{h}\right)\right]-C^{h}\left[\hat{X}^{h}+\sum_{m \in \mathscr{M}}\left(\vec{s}_{m}^{h}-\hat{v}_{m}^{h}\right)\right] \leq 0$, let

$$
\begin{aligned}
B_{m}= & \sum_{h=1}^{H} S_{m}^{h} p^{h}+\mathscr{K}_{1}^{h} P_{2}\left(A_{2}^{h^{\prime}}, s_{m}^{h}-\widehat{s}_{m}^{h}\right) \\
& +\mathscr{K}_{2}^{h} Q_{3}\left(A_{3}^{h^{\prime}}, s_{m}^{h}-\widehat{s}_{m}^{h}\right)+\mathscr{K}_{3}^{h} P_{4}\left(A_{4}^{h^{\prime}}, s_{m}^{h}-\widehat{s}_{m}^{h}\right)+\cdots,
\end{aligned}
$$

where $p^{h} / A_{1}^{h^{\prime}}=\mathscr{K}_{1}^{h} / A_{2}^{h^{\prime}} M=\mathscr{K}_{2}^{h} / A_{3}^{h^{\prime}} M^{2}=\mathscr{K}_{3}^{h} / A_{4}^{h^{\prime}} M^{3}=$ $\cdots=\mathscr{K}_{n}^{h} / A_{n+1}^{h}{ }^{\prime} M^{n}=c>0$. And the incentive for more accurate prediction is provided by the additional term $\mathscr{F}\left(v_{m}^{h}-\right.$ $\left.\hat{v}_{m}^{h}\right)^{2}$.

3.3. Profit Guarantee. In some cases where user load profile changes dramatically, $B_{m}>0$ even when the energy storage device is operated optimally in terms of income maximization. To guarantee profit for each energy storage device, our pricing scheme can be extended further. Assume that $B_{m}$ is the amount to be charged for $m \in \mathscr{M}$ in a whole day according to the pricing scheme introduced in previous sections. Let $\max \left(B_{m}^{+}\right)$denote the maximum positive daily charge of all $m \in \mathscr{M}$. If all the daily charges $B_{m} \leq 0$, then $\max \left(B_{m}^{+}\right)=0$. Our new pricing scheme gives

$$
B_{m}^{\prime}=B_{m}-\max \left(B_{m}^{+}\right), \quad \forall m \in \mathscr{M},
$$

where $B_{m}^{\prime}$ is the amount to be charged in the new pricing scheme. Apparently $B_{m}^{\prime} \leq 0$ which guarantees a profit and storage profile of each $m \in \mathscr{M}$ that minimizes $B_{m}^{\prime}$ is exactly the same as the profile minimizing $B_{m}$. Thus the change in pricing scheme only affects revenue of each energy storage device but has no influence on their decision on storage profile.

\section{Simulation Results}

In this section, we present some simulation results and evaluate the performance of our pricing scheme in different situations. In our simulations, we use the hourly demand data of Ontario, Canada, from the IESO Public Reports [24] for user load profile. Average hourly demand is approximately $15400 \mathrm{MWH}$. Also, we use hourly output data of the 9 wind generators in Ontario for renewable power generation profile. Most of these wind generators have rated hourly output below $150 \mathrm{MWH}$. And we assume that each of these generators is equipped with energy storage device whose charging and discharging volume is $400 \%$ rated power of the generator and has 4 -hour charge/discharge time. We make this assumption to show the performance of our pricing scheme at higher levels of energy storage penetration. In reality economically viable charging and discharging volume and capacity of energy storage device connected with renewable energy source at current stage are much less than the sizes in our assumption. Each energy storage device has charge efficiency $a=0.95$ and discharge efficiency $b=0.95$. At the beginning of each day (also the end of each day), state of charge of

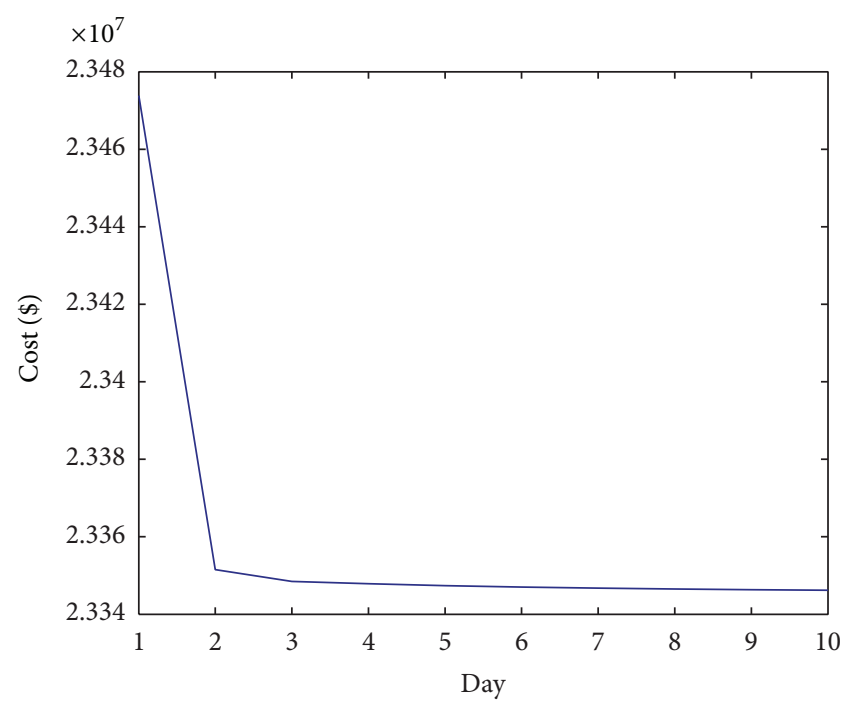

FIGURE 3: Evolution of objective function value (energy generation cost) in the situation where user load and renewable power generation are all constant.

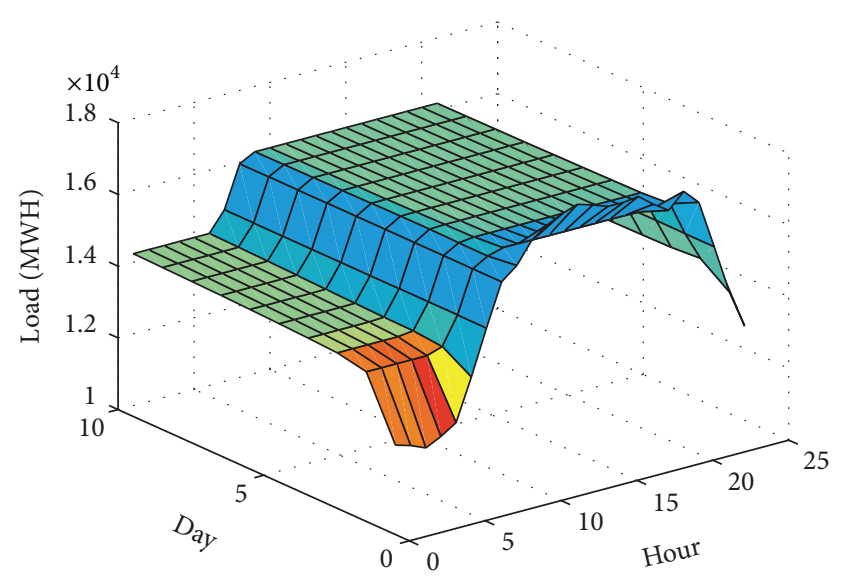

FIGURE 4: Evolution of aggregate demand profile in the situation where both user load and renewable power generation are constant.

each energy storage device is $50 \%$. The objective function (daily energy generation cost) is defined as $\sum_{h=1}^{H} C^{h}\left(l^{h}\right)=$ $\sum_{h=1}^{H} 0.003 l^{h^{2}}+10 l^{h}+100000$.

Simulation results of the objective function value and aggregate demand profile for the situation where user load and renewable power generation keep constant from day to day are shown in Figures 3 and 4. Hourly demand data of Ontario on September 1, 2009, are used as the constant user load profile and hourly output of the 9 wind generators on September 1, 2009, the constant renewable power generation profile. On the first day $s_{m}^{h}=0$ for all $m \in \mathscr{M}$ for all $h \in \mathscr{H}$. Figure 5 compares the aggregate demand profile without energy storage to optimal aggregate demand profile with energy storage that is solved in a centralized manner. From Figures 4 and 5, it can be observed that, under our pricing scheme, aggregate demand profile converges to the optimal profile. 


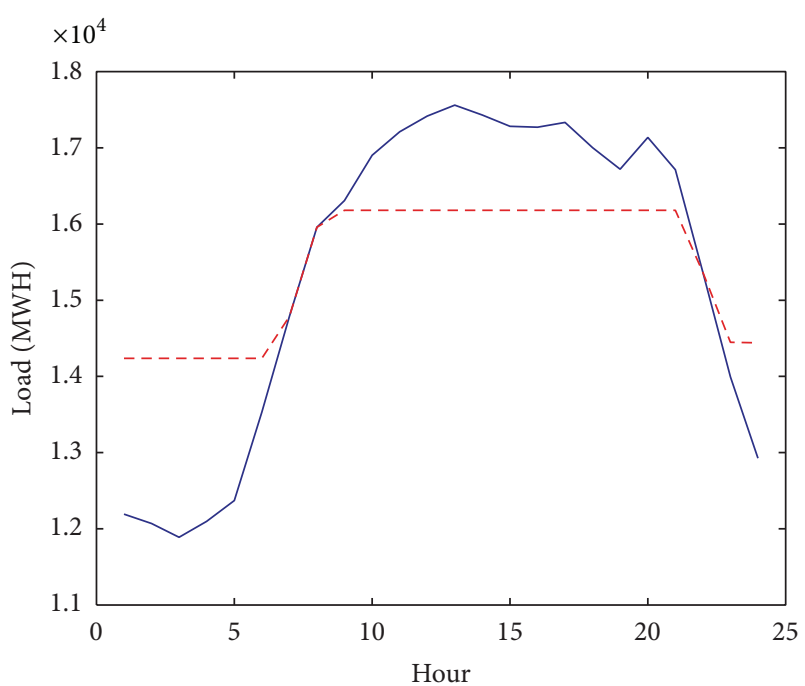

_ Aggregate demand profile without energy storage
- - Optimal profile with energy storage

FIGURE 5: Comparison of aggregate demand profile without energy storage to optimal aggregate demand profile with energy storage.

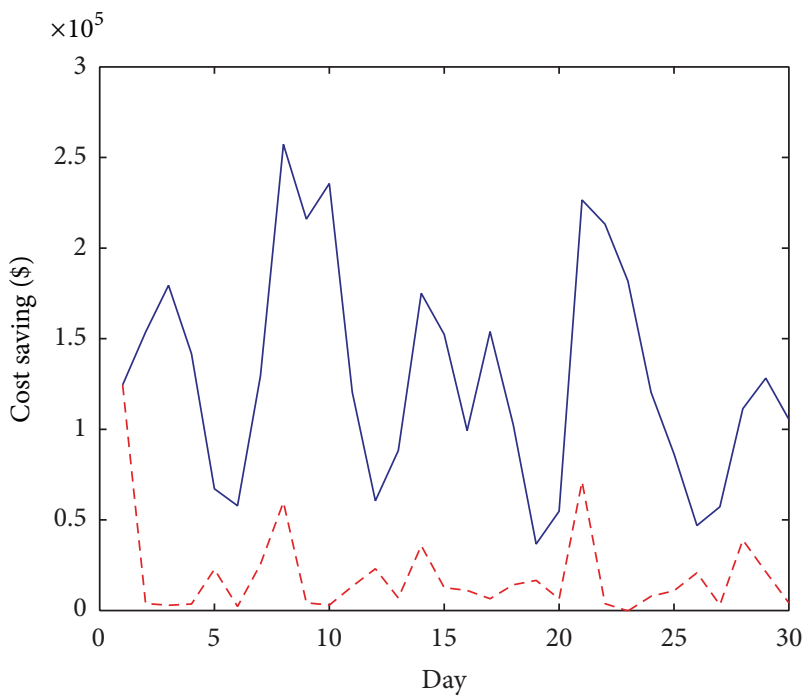

FIGURE 6: Evolution of cost saving: blue line shows cost difference between no energy storage participation and with energy storage participation under our pricing scheme; red line shows cost saved by energy storage changes made to previous day storage profile under our pricing scheme.

For the situation where both user load and renewable power generation are changing, simulation results are shown in Figures 6, 7, and 8. We use hourly demand data of Ontario and hourly output of the 9 wind generators in September 2009 for our simulation. Predicted user load profile and renewable power generation profile are exactly user demand profile and generator output profile in the next day. On August 31, 2009, $s_{m}^{h}=0$ for all $m \in \mathscr{M}$ for all $h \in \mathscr{H}$. It can be observed from Figure 6 that the value of the objective function is reduced every day either compared with the situation where

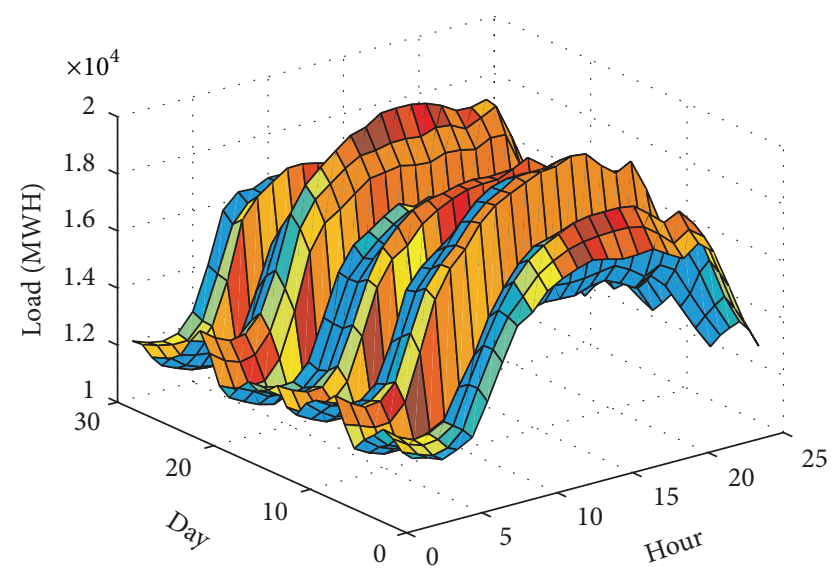

FIGURE 7: Evolution of aggregate demand profile without energy storage in the situation where both user load and renewable power generation are changing.

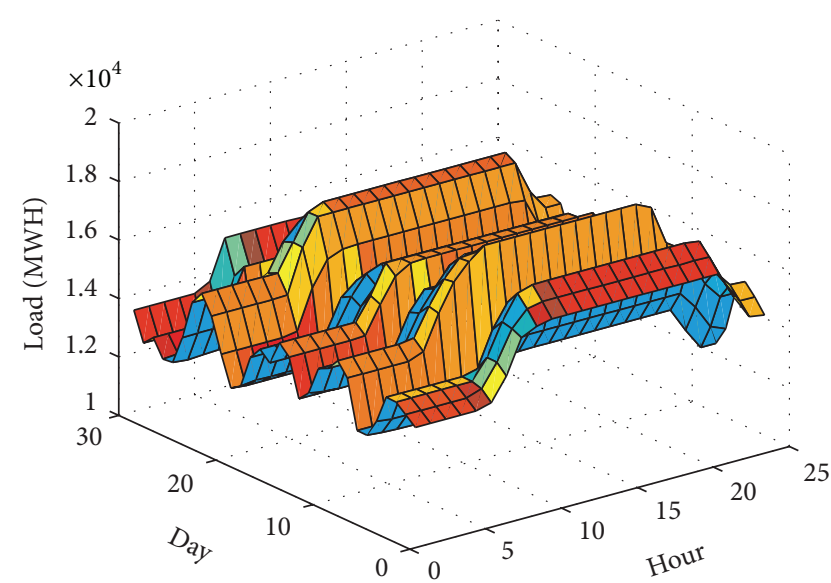

FIGURE 8: Evolution of aggregate demand profile with energy storage in the situation where both user load and renewable power generation are changing.

no energy storage is used or if previous day storage profile is kept. And by comparing Figures 7 and 8, we can see that the aggregate demand profile is efficiently flattened. Ideally, as shown in Figure 9, with ideal charge and discharge efficiency, sufficient charging, and discharging volume as well as energy storage capacity, fully flattened aggregate demand profile can be achieved every day.

However, prediction error is inevitable in reality. As is shown in Figure 7, the shape of daily aggregate demand profile without energy storage is similar from day to day, but the magnitude varies significantly. Therefore what grid operator needs to predict every day is mainly the magnitude of daily aggregate demand profile without energy storage. We now introduce errors into the prediction, which yield normal distribution. If error $\varepsilon_{p}$ is incurred, the predicted aggregate demand profile without energy storage in the coming day will be $\left(1+\varepsilon_{p}\right)\left(\dot{X}^{h}-\sum_{m \in \mathscr{M}} \hat{v}_{m}^{h}\right)$ where $\dot{X}^{h}$ and $\hat{v}_{m}^{h}$ are true total user load profile and renewable energy generation in the next day, respectively. $\varepsilon_{p}$ yields normal distribution whose mean is 0 . 


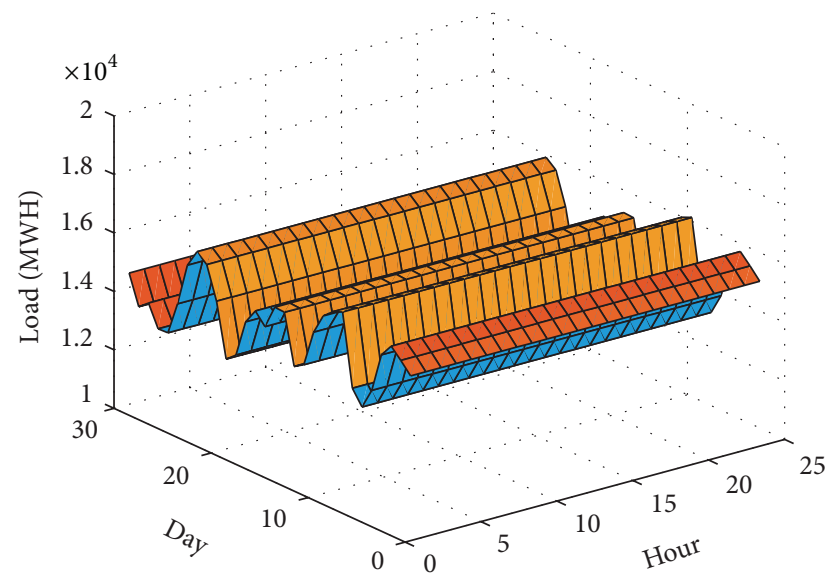

FIGURE 9: Evolution of aggregate demand profile with ideal efficiency, sufficient charging, and discharging volume as well as energy storage capacity.

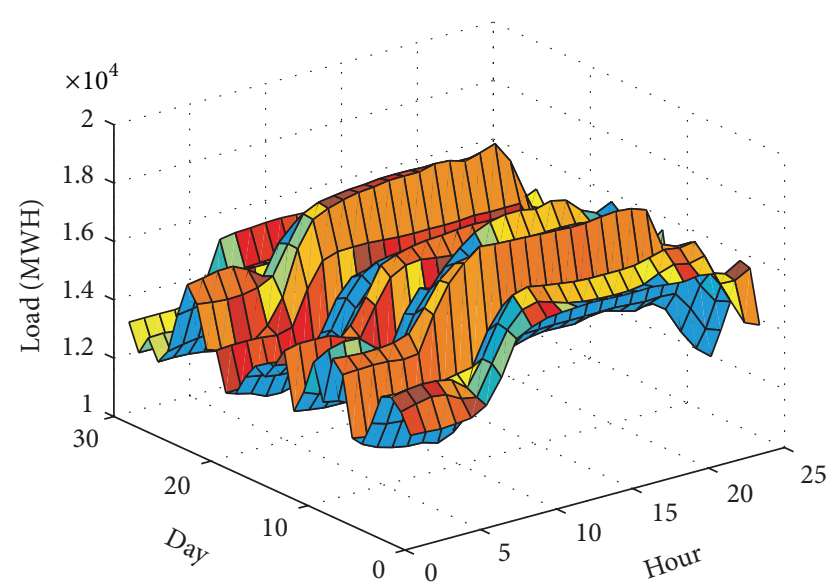

(a)

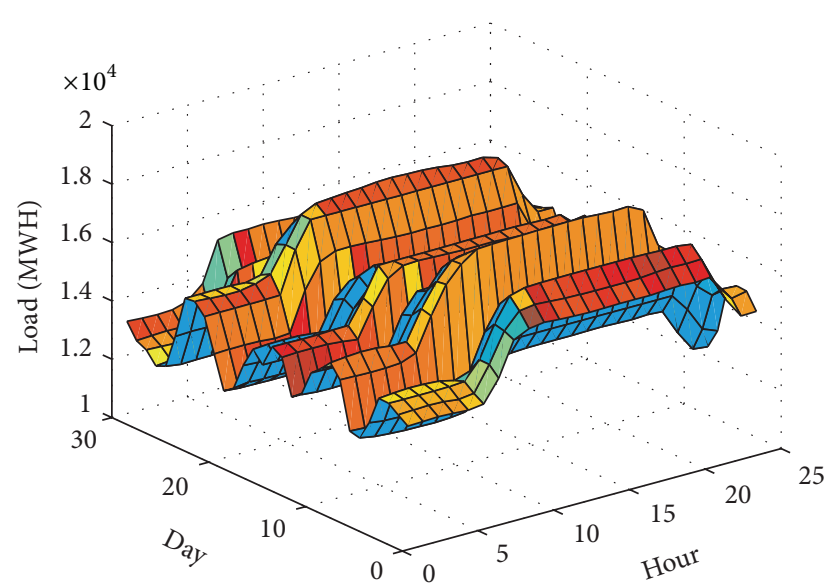

(b)

FIGURE 10: Evolution of aggregate demand profile when standard deviation of the normal distribution $\varepsilon_{p}$ is equal to (a) 0.4 or (b) 0.1 .

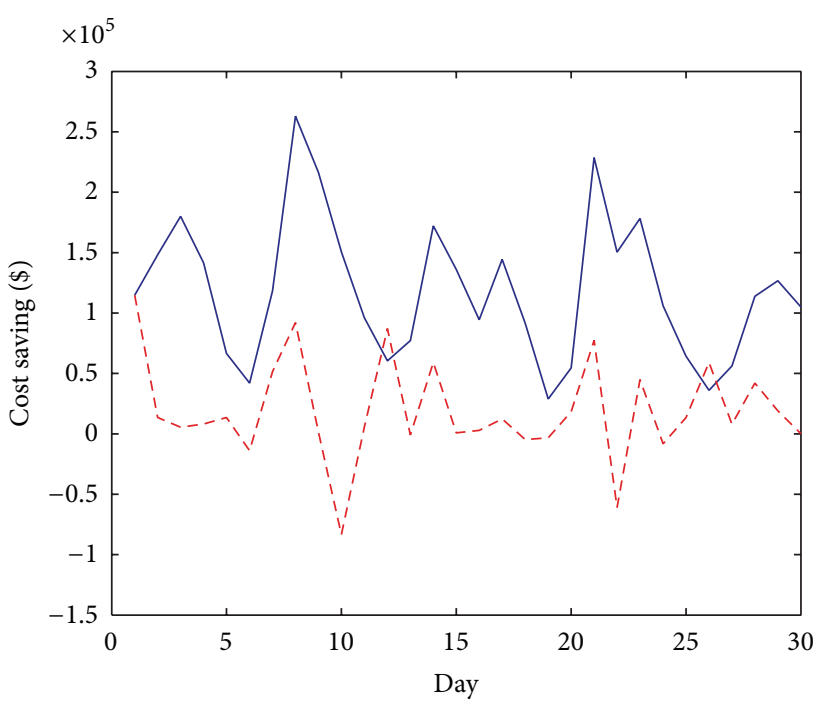

(a)

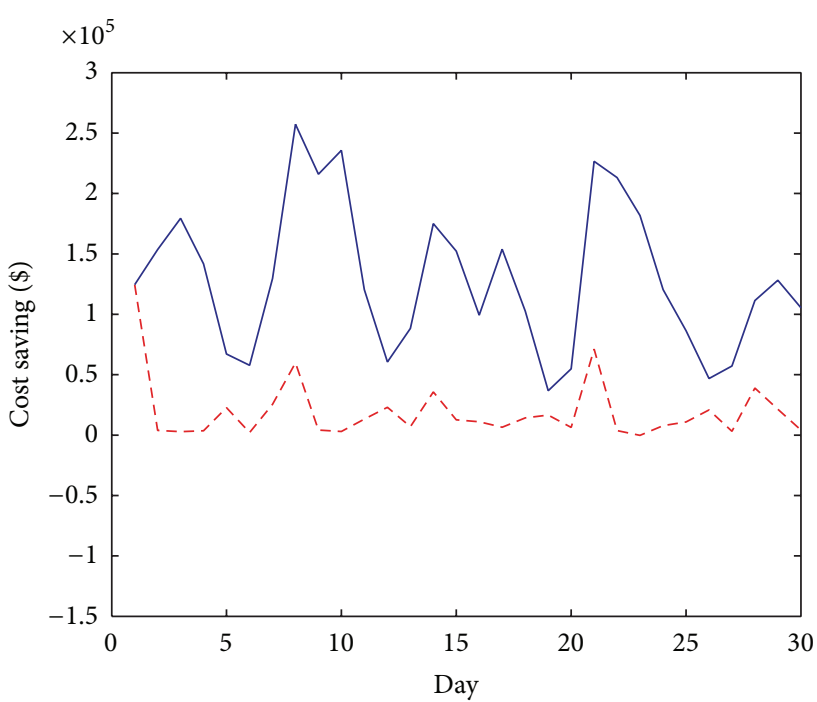

(b)

FIGURE 11: Evolution of cost saving when standard deviation of the normal distribution $\varepsilon_{p}$ is equal to (a) 0.4 or (b) 0.1 ; blue line shows cost difference between no energy storage participation and with energy storage participation under our pricing scheme; red line shows cost saved by energy storage changes made to previous day storage profile under our pricing scheme.

Evolution of aggregate demand profile and cost saving in the situations where standard deviation of the normal distribution $\varepsilon_{p}$ is equal to 0.1 or 0.4 is shown in Figures 10 and 11 .

After prediction errors are introduced, profitability of our pricing scheme can not be guaranteed when prediction errors are too large which is rare but possible (Figure 11). But in general, our pricing scheme still reduces the value of objective function efficiently and the more accurate the predictions are, the better the objectives are met. 


\section{Conclusion}

In this paper, a universal pricing scheme was proposed to indirectly control energy storage devices in smart grid. It was designed to efficiently reduce the value of any convex objective function defined by grid operators. We proved that, in the situation where user load and renewable energy generation profile keep constant and each energy storage device is operated optimally in terms of income maximization, aggregate demand profile is convergent to the optimal profile which minimizes the convex objective function under our pricing scheme. When both user load and renewable energy generation are changing from day to day, our pricing scheme can still efficiently reduce the value of the objective function, which can satisfactorily meet particular targets of grid operators. Profitability of optimal energy storage operation can also be guaranteed. Simulation results assuming high level of energy storage penetration were provided to demonstrate the stability and profitability of our pricing scheme. Our pricing scheme can be applied to control the behavior of energy storage devices installed for integration of intermittent renewable energy at current stage and is believed to have much broader applications in the future.

\section{Appendices}

\section{A. Proof of Theorem 1}

If device $m$ does not change its storage profile in the coming day, $B_{m}\left(\widetilde{s}_{m}\right)=\sum_{h=1}^{H} p^{h}\left(\widetilde{s}_{m}^{h}-v_{m}^{h}\right)$, where $\widetilde{s}_{m}$ is the storage profile of $m$ in the day before.

Assume that the new storage profile $s_{m}=\widetilde{s}_{m}+\Delta s_{m}$ is adopted. If device $m$ is operated optimally in terms of income maximization, $\Delta s_{m}$ will at least keep $B_{m}$ the same or possibly decrease $B_{m}$. Consider

$$
\begin{gathered}
B_{m}\left(\widetilde{s}_{m}+\Delta s_{m}\right)=\sum_{h=1}^{H} p^{h}\left(\widetilde{s}_{m}^{h}+\Delta s_{m}^{h}-v_{m}^{h}\right)+\mathscr{K} \sum_{h=1}^{H} \Delta s_{m}^{h^{2}}, \\
B_{m}\left(\widetilde{s}_{m}+\Delta s_{m}\right)-B_{m}\left(\widetilde{s}_{m}\right)=\sum_{h=1}^{H} p^{h} \Delta s_{m}^{h}+\mathscr{K} \sum_{h=1}^{H} \Delta s_{m}^{h^{2}} \leq 0, \\
\sum_{m \in \mathscr{M}}\left(\sum_{h=1}^{H} p^{h} \Delta s_{m}^{h}+\mathscr{K} \sum_{h=1}^{H} \Delta s_{m}^{h^{2}}\right) \leq 0 .
\end{gathered}
$$

By definition $l^{h}=\sum_{m \in \mathscr{M}}\left(s_{m}^{h}-v_{m}^{h}\right)+\sum_{n \in \mathcal{N}} x_{n}^{h}$ and $\Delta l^{h}=$ $\sum_{m \in \mathscr{M}} \Delta s_{m}^{h}$,

$$
\Delta l^{h^{2}} \leq M \sum_{m \in M} \Delta s_{m}^{h^{2}}
$$

where $M$ is the total number of energy storage devices, which implies

$$
\begin{aligned}
& \sum_{h=1}^{H} p^{h} \Delta l^{h}+\frac{\mathscr{K}}{M} \sum_{h=1}^{H} \Delta l^{h^{2}} \\
& \quad \leq \sum_{h=1}^{H} p^{h} \sum_{m \in \mathscr{M}} \Delta s_{m}^{h}+\mathscr{K} \sum_{h=1}^{H} \sum_{m \in \mathscr{M}} \Delta s_{m}^{h^{2}} \leq 0 .
\end{aligned}
$$

We have $p^{h}=\mathscr{K}\left(2 a_{k} \widetilde{l}^{h}+b_{k}\right) / a_{k} M$; then

$$
\begin{aligned}
& \sum_{h=1}^{H} \frac{\mathscr{K}\left(2 a_{k} \tilde{l}^{h}+b_{k}\right)}{a_{k} M} \Delta l^{h}+\frac{\mathscr{K}}{M} \sum_{h=1}^{H} \Delta l^{h^{2}} \leq 0, \\
& \sum_{h=1}^{H}\left(2 a_{k} \tilde{l}^{h}+b_{k}\right) \Delta l^{h}+a_{k} \sum_{h=1}^{H} \Delta l^{h^{2}} \leq 0, \\
& \sum_{h=1}^{H} C^{h}\left(\tilde{l}^{h}+\Delta l^{h}\right)-C^{h}\left(\tilde{l}^{h}\right) \\
& =\sum_{h=1}^{H} 2 a_{k} \tilde{l}^{h} \Delta l^{h}+a_{k} \Delta l^{h^{2}}+b_{k} \Delta l^{h} \leq 0,
\end{aligned}
$$

where $\widetilde{l}^{h}+\Delta l^{h}$ is aggregate demand profile in the coming day and the value of the objective function is reduced or kept the same with the new profile.

\section{B. Proof of Theorem 2}

Assume that $\tilde{l}^{h}$ is the current aggregate demand profile, $l^{h^{\prime}}$ the optimal aggregate demand profile, which minimizes the objective function, and $s_{m}^{h{ }^{\prime}}$ the corresponding storage profile of each device $m$ when optimal aggregate demand profile is achieved. Consider

$$
\begin{gathered}
\sum_{h=1}^{H} C^{h}\left(l^{h^{\prime}}\right)-C^{h}\left(\widetilde{l}^{h}\right)=\epsilon<0 . \\
\text { Let } \lambda^{h}=l^{h^{\prime}}-\widetilde{l}^{h}=\sum_{m \in \mathscr{M}}\left(s_{m}^{h^{\prime}}-\widetilde{s}_{m}^{h}\right) \\
\sum_{h=1}^{H} 2 a_{k} \widetilde{l}^{h} \lambda^{h}+a_{k} \lambda^{h^{2}}+b_{k} \lambda^{h}=\epsilon, \\
\sum_{h=1}^{H} p^{h} \lambda^{h}+\frac{\mathscr{K}}{M} \lambda^{h^{2}}=\epsilon \frac{\mathscr{K}}{a_{k} M}, \\
\sum_{h=1}^{H} p^{h} \lambda^{h} \leq \epsilon \frac{\mathscr{K}}{a_{k} M} .
\end{gathered}
$$

Therefore, $\exists m$ such that $\sum_{h=1}^{H} p^{h} \Delta \check{s}_{m}^{h}=\epsilon^{\prime}<0$, where $\Delta \check{s}_{m}^{h}=s_{m}^{h^{\prime}}-\widehat{s}_{m}^{h}$.

Let $\Delta \widehat{s}_{m}^{h}$ denote the valid $\Delta s_{m}^{h}$ which minimizes $\sum_{h=1}^{H} p^{h} \Delta s_{m}^{h}+\mathscr{K} \sum_{h=1}^{H} \Delta s_{m}^{h^{2}}$. As storage profile converges, for 
all $\varepsilon>0$ there exists a day such that the absolute value of $\Delta \widehat{s}_{m}^{h}$ after this day is smaller than $\varepsilon$. Consider

$$
\left|\sum_{h=1}^{H} p^{h} \Delta \widehat{s}_{m}^{h}+\mathscr{K} \sum_{h=1}^{H} \Delta \widehat{s}_{m}^{h^{2}}\right| \leq \sum_{h=1}^{H}\left|p^{h}\right| \varepsilon+\mathscr{K} H \varepsilon^{2} .
$$

Thus,

$$
\begin{aligned}
\min & \left(\sum_{h=1}^{H} p^{h} \Delta s_{m}^{h}+\mathscr{K} \sum_{h=1}^{H} \Delta s_{m}^{h^{2}}\right) \\
& =\sum_{h=1}^{H} p^{h} \Delta \hat{s}_{m}^{h}+\mathscr{K} \sum_{h=1}^{H} \Delta \widehat{s}_{m}^{h^{2}} \geq-\sum_{h=1}^{H}\left|p^{h}\right| \varepsilon-\mathscr{K} H \varepsilon^{2} .
\end{aligned}
$$

For each $m \in \mathscr{M}$, which satisfies $\sum_{h=1}^{H} p^{h} \Delta \check{s}_{m}^{h}=\epsilon^{\prime}<$ $0, \exists \alpha_{0}=-\epsilon^{\prime} / \mathscr{K} \sum_{h=1}^{H} \Delta \check{s}_{m}^{h^{2}}>0$ such that $\sum_{h=1}^{H} p^{h} \Delta \check{s}_{m}^{h}+$ $\mathscr{K} \alpha_{0} \sum_{h=1}^{H} \Delta \check{s}_{m}^{h}=0$. If $\alpha_{0} \geq 1$, let $\Delta s_{m}^{h}=0.5 \Delta \check{s}_{m}^{h}$ so that $\Delta s_{m}^{h}$ is surely valid. $\sum_{h=1}^{H} p^{h} \Delta s_{m}^{h}+\mathscr{K} \sum_{h=1}^{H} \Delta s_{m}^{h^{2}}=(0.25-$ $\left.0.5 \alpha_{0}\right) \mathscr{K} \sum_{h=1}^{H} \Delta \check{s}_{m}^{h}$. Or if $\alpha_{0}<1$, let $\Delta s_{m}^{h}=0.5 \alpha_{0} \Delta \check{s}_{m}^{h}$, $\sum_{h=1}^{H} p^{h} \Delta s_{m}^{h}+\mathscr{K} \sum_{h=1}^{H} \Delta s_{m}^{h^{2}}=-\left(\alpha_{0}^{2} / 4\right) \mathscr{K} \sum_{h=1}^{H} \Delta \check{s}_{m}^{h^{2}}$. Thus,

$$
\begin{aligned}
\min \left(\sum_{h=1}^{H} p^{h} \Delta s_{m}^{h}+\mathscr{K} \sum_{h=1}^{H} \Delta s_{m}^{h^{2}}\right) & \\
& \leq \begin{cases}-\frac{\alpha_{0}^{2}}{4} \mathscr{K} \sum_{h=1}^{H} \Delta \check{s}_{m}^{h^{2}}, & 0<\alpha_{0}<1 ; \\
\left(0.25-0.5 \alpha_{0}\right) \mathscr{K} \sum_{h=1}^{H} \Delta \check{s}_{m}^{h}, & \alpha_{0} \geq 1 .\end{cases}
\end{aligned}
$$

As a result,

$$
\begin{aligned}
\sum_{h=1}^{H}\left|p^{h}\right| & \varepsilon+\mathscr{K} H \varepsilon^{2} \\
\geq & \begin{cases}\frac{\alpha_{0}^{2}}{4} \mathscr{K} \sum_{h=1}^{H} \Delta \check{s}_{m}^{h}, & 0<\alpha_{0}<1 ; \\
\left(-0.25+0.5 \alpha_{0}\right) \mathscr{K} \sum_{h=1}^{H} \Delta \check{s}_{m}^{h^{2}}, & \alpha_{0} \geq 1 .\end{cases}
\end{aligned}
$$

If $\alpha_{0} \rightarrow 0$, then $\epsilon^{\prime} \rightarrow 0$ for each $m \in \mathscr{M}$ satisfying $\sum_{h=1}^{H} p^{h} \Delta \check{s}_{m}^{h}=\epsilon^{\prime}<0$ since $0 \leq \mathscr{K} \sum_{h=1}^{H} \Delta \check{s}_{m}^{h}<\mathscr{K} H\left(s_{+}^{2}+s_{-}^{2}\right)$. Otherwise, $\Delta \check{s}_{m}^{h}$ converges to 0 and so is $\epsilon^{\prime}$ for each $m \in \mathscr{M}$ satisfying $\sum_{h=1}^{H} p^{h} \Delta \check{s}_{m}^{h}=\epsilon^{\prime}<0$. Since $\sum_{h=1}^{H} \sum_{m \in M} p^{h} \Delta \check{s}_{m}^{h}=$ $\sum_{h=1}^{H} p^{h} \lambda^{h} \leq \epsilon\left(\mathscr{K} / a_{k} M\right)<0, \epsilon$ converges to 0 .

\section{Proof of Theorem 3}

Assume new storage profile $s_{m}=\widetilde{s}_{m}+\Delta s_{m}$ is adopted, where $\widetilde{s}_{m}$ is storage profile of $m$ in the day before. Then,

$$
\begin{gathered}
B_{m}\left(\widetilde{s}_{m}+\Delta s_{m}\right)-B_{m}\left(\tilde{s}_{m}\right) \\
=\sum_{h=1}^{H} \Delta s_{m}^{h} p^{h}+\mathscr{K}_{1}^{h} P_{2}\left(A_{2}^{h}, \Delta s_{m}^{h}\right) \\
\quad+\mathscr{K}_{2}^{h} Q_{3}\left(A_{3}^{h}, \Delta s_{m}^{h}\right)+\mathscr{K}_{3}^{h} P_{4}\left(A_{4}^{h}, \Delta s_{m}^{h}\right)+\cdots \leq 0, \\
\sum_{h=1}^{H} C^{h}\left(\widetilde{l}^{h}+\Delta l^{h}\right)-C^{h}\left(\tilde{l}^{h}\right) \\
\leq \sum_{h=1}^{H} A_{1}^{h} \Delta l^{h}+A_{2}^{h} P_{2}\left(A_{2}^{h}, \Delta l^{h}\right) \\
\quad+A_{3}^{h} Q_{3}\left(A_{3}^{h}, \Delta l^{h}\right)+A_{4}^{h} P_{4}\left(A_{4}^{h}, \Delta l^{h}\right)+\cdots \\
\leq \sum_{h=1}^{H} A_{1}^{h} \sum_{m \in \mathscr{M}} \Delta s_{m}^{h}+A_{2}^{h} M \sum_{m \in \mathscr{M}} P_{2}\left(A_{2}^{h}, \Delta s_{m}^{h}\right) \\
\quad+A_{3}^{h} M^{2} \sum_{m \in \mathscr{M}} Q_{3}\left(A_{3}^{h}, \Delta s_{m}^{h}\right) \\
\quad+A_{4}^{h} M^{3} \sum_{m \in \mathscr{M}} P_{4}\left(A_{4}^{h}, \Delta s_{m}^{h}\right)+\cdots \leq 0 .
\end{gathered}
$$

This completes the proof.

\section{Conflict of Interests}

The authors declare that there is no conflict of interests regarding the publication of this paper.

\section{Acknowledgments}

This work is supported by the National Natural Science Foundation of China (NNSFC) under Grants 61374053 and 61104118, HKU CRCG 201111159110, and the Zijin Intelligent Program of Nanjing University of Science and Technology.

\section{References}

[1] S. M. Amin and B. F. Wollenberg, "Toward a smart grid," IEEE Power and Energy Magazine, vol. 3, no. 5, pp. 34-41, 2005.

[2] U. S. Department of Energy, The Smart Grid: An Introduction, 2009.

[3] M. Pipattanasomporn, H. Feroze, and S. Rahman, "Multi-agent systems in a distributed smart grid: Design and implementation," in Proceedings of the IEEE/PES Power Systems Conference and Exposition (PSCE '09), p. 18, March 2009.

[4] T. Voice, P. Vytelingum, S. Ramchurn, A. Rogers, and N. Jennings, "Decentralised control of micro-storage in the smart grid," in Proceedings of the AAAI Conference on Articial Intelligence, 2011. 
[5] K. T. Tan, X. Y. Peng, P. L. So, Y. C. Chu, and M. Z. Q. Chen, "Centralized control for parallel operation of distributed generation inverters in microgrids," IEEE Transactions on Smart Grid, vol. 3, no. 4, pp. 1977-1987, 2012.

[6] K. T. Tan, P. L. So, Y. C. Chu, and M. Z. Q. Chen, "Coordinated control and energy management of distributed generation inverters in a microgrid," IEEE Transactions on Power Delivery, vol. 28, no. 2, pp. 704-713, 2013.

[7] K. T. Tan, P. L. So, Y. C. Chu, and M. Z. Q. Chen, "A flexible AC distribution system device for a microgrid," IEEE Transactions on Energy Conversion, vol. 28, no. 3, pp. 601-610, 2013.

[8] Y. Chu and M. Z. Q. Chen, "Efficient model predictive algorithms for tracking of periodic signals," Journal of Control Science and Engineering, vol. 2012, Article ID 729748, 13 pages, 2012.

[9] C. W. Potter, A. Archambault, and K. Westrick, "Building a smarter smart grid through better renewable energy information," in Proceedings of the IEEE/PES Power Systems Conference and Exposition (PSCE '09), pp. 1-5, Seattle, Wash, USA, March 2009.

[10] Z. Gao, D. Kong, and C. Gao, "Modeling and control of complex dynamic systems: applied mathematical aspects," Journal of Applied Mathematics, vol. 2012, Article ID 869792, 5 pages, 2012.

[11] Z. Gao, D. Kong, and C. Gao, "Modeling and control of complex dynamic systems: applied mathematical aspects," Journal of Applied Mathematics, vol. 2012, Article ID 869792, 5 pages, 2012.

[12] C. A. Hill, M. C. Such, D. Chen, J. Gonzalez, and W. M. Grady, "Battery energy storage for enabling integration of distributed solar power generation," IEEE Transactions on Smart Grid, vol. 3, no. 2, pp. 850-857, 2012.

[13] N. Miller, D. Manz, J. Roedel, P. Marken, and E. Kronbeck, "Utility scale battery energy storage systems," in Proceedings of the IEEE Power Engineering Society, IEEE General Meeting, Minneapolis, Minn, USA.

[14] M. Kintner-Meyer, C. Jin, P. Balducci et al., "Energy storage for variable renewable energy resource integration: a regional assessment for the Northwest Power Pool (NWPP)," in Proceeding of the IEEE/PES Power Systems Conference and Exposition (PSCE '11), pp. 1-7, Phoenix, Ariz, USA, March 2011.

[15] N. S. Wade, P. C. Taylor, P. D. Lang, and P. R. Jones, "Evaluating the benefits of an electrical energy storage system in a future smart grid," Energy Policy, vol. 38, no. 11, pp. 7180-7188, 2010.

[16] J. Eyer and G. Corey, "Energy storage for the electricity grid," SANDIA REPORT, Sandia National Labs Publications, 2010, http://prod.sandia.gov/techlib/access-control.cgi/2010/100815 .pdf.

[17] S. D. Ramchurn, P. Vytelingum, A. Rogers, and N. R. Jennings, "Agent-based homeostatic control for green energy in the smart grid," ACM Transactions on Intelligent Systems and Technology, vol. 2, no. 4, article 35, 2011

[18] P. Vytelingum, T. D. Voice, S. D. Ramchurn, A. Rogers, and N. R. Jennings, "Agent-based micro-storage management for the smart grid," in Proceedings of the 9th International Conference on Autonomous Agents and Multiagent Systems, pp. 39-46, 2010.

[19] S. Teleke, "Energy Storage Overview: Applications, Technologies and Economical Evaluation," Quanta Technology Technical Papers, http://www.sandia.gov/ess/publications/SAND20100815.pdf.

[20] A. Mohsenian-Rad, V. W. S. Wong, J. Jatskevich, R. Schober, and A. Leon-Garcia, "Autonomous demand-side management based on game-theoretic energy consumption scheduling for the future smart grid," IEEE Transactions on Smart Grid, vol. 1, no. 3, pp. 320-331, 2010.

[21] C. Wu, H. Mohsenian-Rad, J. Huang, and A. Y. Wang, "Demand side management for wind power integration in microgrid using dynamic potential game theory," in Proceedings of the IEEE GLOBECOM Workshop on Smart Grid Communications and Networking, Houston, Tex, USA, 2011.

[22] A. Mohsenian-Rad and A. Leon-Garcia, "Optimal residential load control with price prediction in real-time electricity pricing environments," IEEE Transactions on Smart Grid, vol. 1, no. 2, pp. 120-133, 2010.

[23] P. Samadi, H. Mohsenian-Rad, R. Schober, V. Wong, and J. Jatskevich, "Optimal real-time pricing algorithm ba sed on utility maximization for smart grid," in Proceedings of the IEEE Conference on Smart Grid Communications, 2010.

[24] IESO Public Reports, http://reports.ieso.ca/public/. 


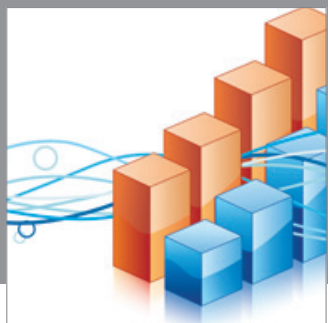

Advances in

Operations Research

mansans

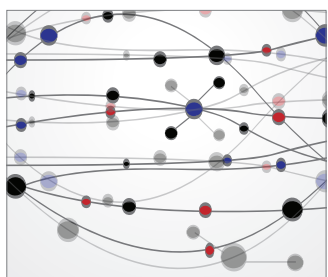

The Scientific World Journal
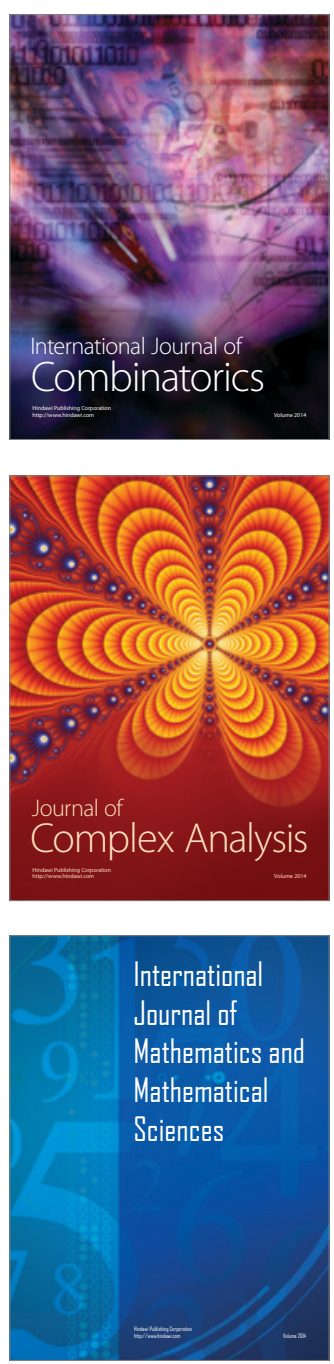
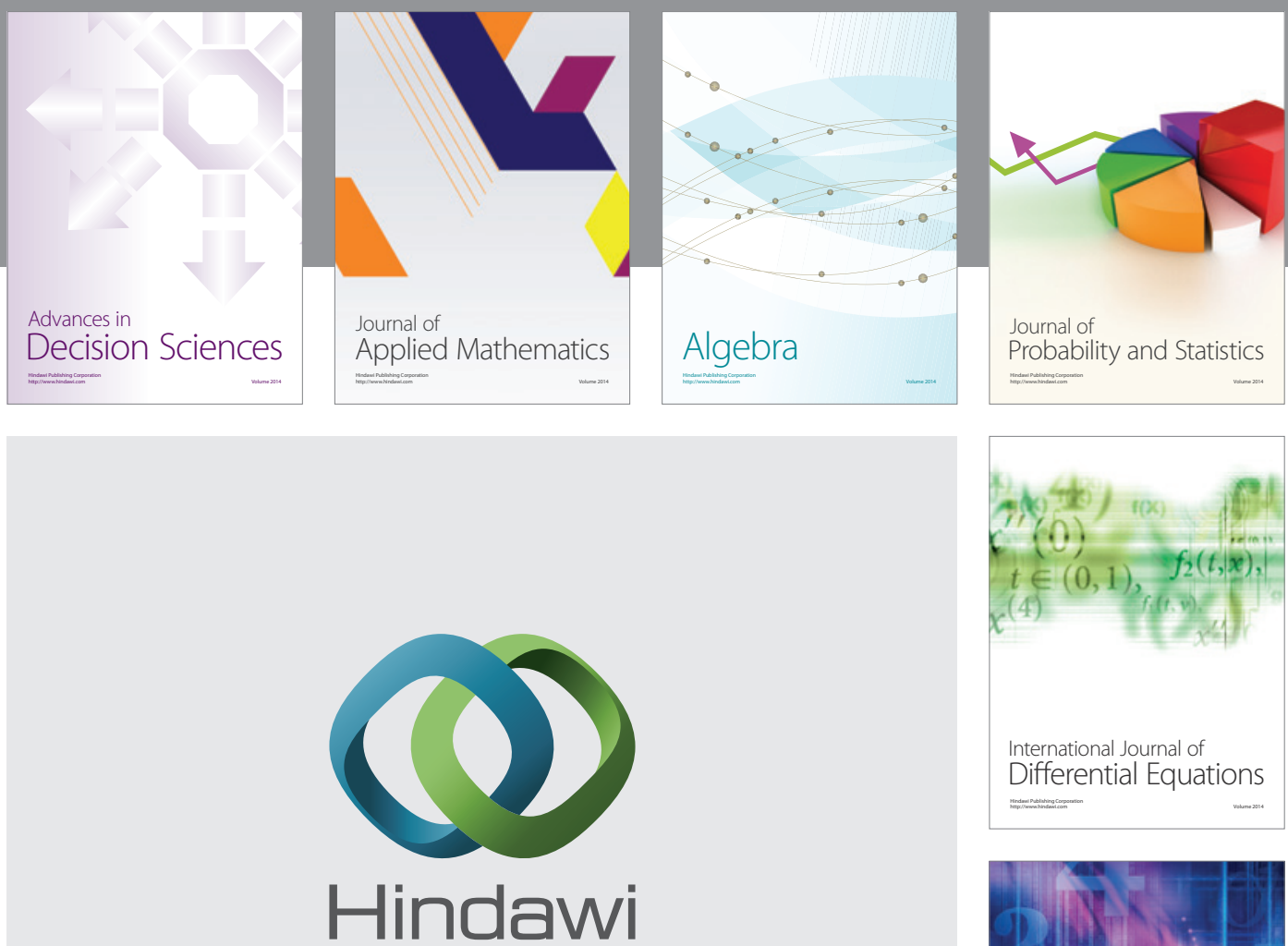

Submit your manuscripts at http://www.hindawi.com
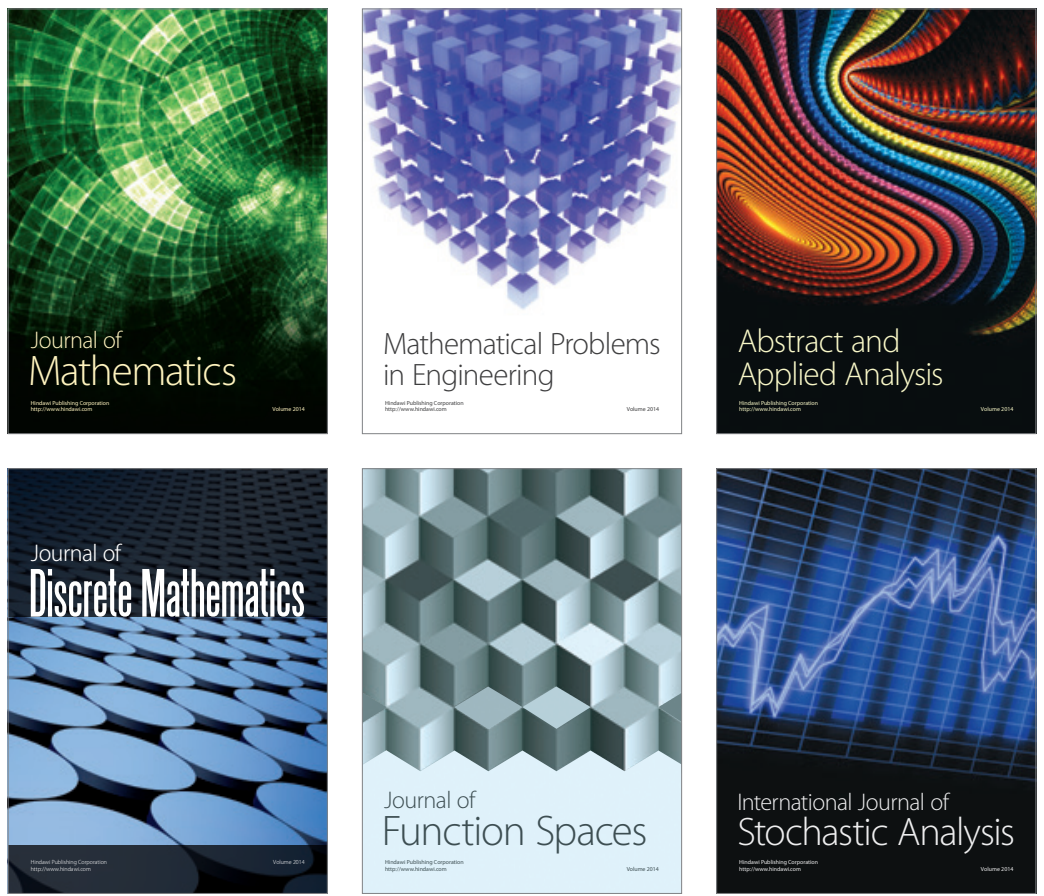

Journal of

Function Spaces

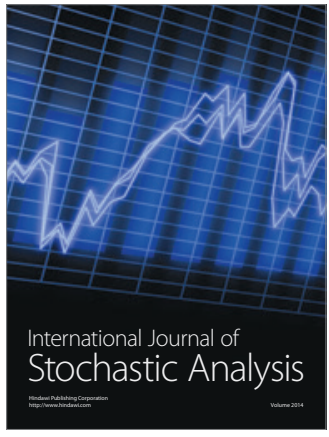

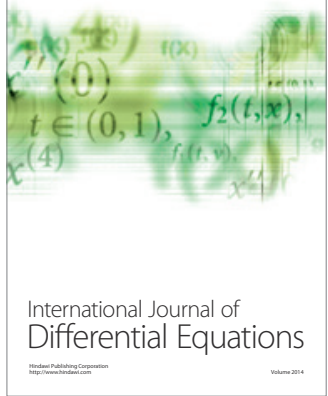
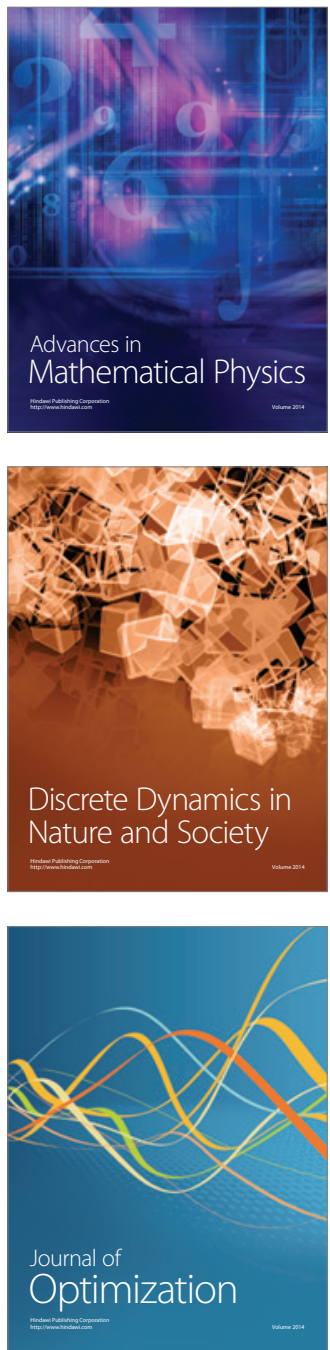